\title{
ON THE BV STRUCTURE ON THE COHOMOLOGY OF MODULI SPACE
}

\author{
SÜMEYRA SAKALLI AND ALEXANDER A. VORONOV \\ Dedicated to Kyoji Saito on the occasion of his 75th birthday
}

\begin{abstract}
The question of vanishing of the BV operator on the cohomology of the moduli space of Riemann surfaces is investigated. The BV structure, which comprises a BV operator and an antibracket, is identified, vanishing theorems are proven, and a counterexample is provided.
\end{abstract}

\section{INTRODUCTION}

Since the early nineties, there has been considerable interest in nonperturbative methods in string field theory, in particular, setting up a quantum master equation $(Q M E)$

$$
d S+\hbar \Delta S+\frac{1}{2}\{S, S\}=0
$$

in the dg-BV-algebra $C_{c}^{\bullet}(\mathcal{M})$ of compactly supported cochains with rational coefficients on the moduli space $\mathcal{M}$ of Riemann surfaces and describing a solution $S$ of the QME, see, for example, [44, 45, 39, 24, 10, 30, 6, 7, 16, 22, 20, 32, 23, 9, 5]. One work that caught the eye was that of Kevin Costello [7], in which he used homotopical-algebraic methods and certain elementary facts about the cohomology of moduli spaces to prove the existence and uniqueness, up to homotopy, of a solution $S$ of the QME. The proof consisted in a clever reduction of the QME to a linear equation

$$
\hat{d} S^{\prime}=0
$$

on a related $S^{\prime}$ in a homotopy abelian subquotient of $C_{c}^{\bullet}(\mathcal{M})$ for the differential $\hat{d}=d+\hbar \Delta$ and transferring the problem to cohomology.

Date: December 29, 2019.

2010 Mathematics Subject Classification. 14H15 (Primary) 14F17, 32G15 (Secondary). 
Note that the QME (1) is actually a Maurer-Cartan equation

$$
\hat{d} S+\frac{1}{2}\{S, S\}=0
$$

in the dg-Lie algebra of cochains on the moduli space and if this dg-Lie algebra is homotopy abelian, then the Maurer-Cartan equation is equivalent to the cocycle equation (2). It is known, see [40, 21], that in a dg-BValgebra, the homotopy abelianness of the underlying dg-Lie algebra may be derived from the degeneration at $E_{1}$ of a spectral sequence associated to the double complex $\left(C_{c}^{\bullet}(\mathcal{M}), d, \Delta\right)$, which entails the vanishing of the differential $d_{1}=\Delta$ on the cohomology $H_{c}^{\bullet}(\mathcal{M})$.

Given Costello's homotopy abelianness result, one might hope that homotopy abelianness takes place for the whole dg-Lie algebra $C_{c}^{\bullet}(\mathcal{M})$ and that the general argument of [40, 21] would imply Costello's existence and uniqueness result. On the other hand, any general statement, such as the vanishing of a cohomology operation, $\Delta$ in particular, on the cohomology of the moduli space is extremely interesting, given how little is still known about it.

This paper grew out of our investigation of the vanishing of $\Delta$ on the cohomology $H_{c}^{\bullet}(\mathcal{M})$ of the moduli space. It has turned out that $\Delta$ vanishes on vast ranges of these cohomology groups, appearing to provide convincing evidence for vanishing everywhere, see Section 2 below. On the other hand, we have found out that total vanishing does not actually take place. We provide a counterexample in Corollary 2.7. In the process, we obtain useful description of the BV operator $\Delta$ and the antibracket $\{-,-\}$, relating them to the differential $d_{1}$ in the spectral sequence associated to the topological filtration on the compactified moduli space $\overline{\mathcal{M}}$. This differential may be expressed as the Gysin homomorphism given by Poincaré residue, see Section 1.3. It is somewhat surprising that the differentials on the first terms of the spectral sequences associated to the double complex $\left(C_{c}^{\bullet}(\mathcal{M}), d, \Delta\right)$ and the topological filtration on $\overline{\mathcal{M}}$ are closely related, see Theorem 1.2 .

Conventions. In this paper, $\mathcal{M}_{g, n}$ denotes the (usual) moduli space of smooth, connected, compact, genus $g$ Riemann surfaces with $n$ labeled punctures. Here and throughout the paper, we assume that our Riemann 
surfaces are stable, i.e., $n \geq 3$ if $g=0$ and $n \geq 1$ if $g=1$; otherwise $g, n \geq 0$. Let $S_{n}$ be the group of permutations of $n$ objects. Then $\mathcal{M}_{g}(n):=\mathcal{M}_{g, n} / S_{n}$ is the moduli space of compact Riemann surfaces with $n$ unlabeled punctures.

We work with homology $H_{\bullet}(X):=H_{\bullet}(X, \mathbb{Q})$ and cohomology $H^{\bullet}(X):=$ $H^{\bullet}(X, \mathbb{Q})$ with rational coefficients. Vector spaces are also assumed to be over $\mathbb{Q}$.

Acknowledgments. With admiration and gratitude, we dedicate this paper to Kyoji Saito. The breadth of his interests touched upon the topics of this paper as well: his recent paper [26] uses the BV-algebra of multivector fields on a Calabi-Yau manifold, whereas his interest in the moduli spaces is long-standing, see [35, 36, 37, 38]. We are grateful to Weiyan Chen, Domenico D'Alessandro, Søren Galatius, and Craig Westerland for useful discussions. SS also thanks the Max Planck Institute for Mathematics, Bonn, for its great research environment and support. AV was supported by the World Premier International Research Center Initiative (WPI), MEXT, Japan, and a Collaboration grant from the Simons Foundation (\#585720).

\section{The BV STRUCTURE ON THE (CO)HOMOLOGY OF MODUli SPACES}

1.1. Operations on the homology $H_{\bullet}\left(\mathcal{M}_{g}(n), \mathbb{Q}\right)$. The following operations have been originally defined by Zwiebach [45] and modified by Costello [7].

1. For $n \geq 0$, define the $B V$ operator

$$
\Delta: H_{k}\left(\mathcal{M}_{g}(n+2)\right) \rightarrow H_{k+1}\left(\mathcal{M}_{g+1}(n)\right)
$$

as follows. If $Z \in H_{k}\left(\mathcal{M}_{g}(n+2)\right)$ is a homology class represented by a singular cycle $Z=\sum_{i=1}^{m} c_{i} \sigma_{i}$ for $\sigma_{i}: \Delta^{k} \rightarrow \mathcal{M}_{g}(n+2)$, then set

$$
\left.\Delta(Z):=\sum_{i} c_{i} \sum_{\{\beta, \gamma\}} \text { (twist-gluing of } \sigma_{i} \text { at punctures }\{\beta, \gamma\}\right),
$$

where the summation runs over unordered pairs $\{\beta, \gamma\}$ of punctures and twist-gluing is cutting out small holomorphic disks around the punctures 
$\{\beta, \gamma\}$, and gluing their complement(s) along the boundaries. More precisely, we consider holomorphic disks at each puncture (i.e., holomorphic embeddings of the standard disk $|z|<1$ centered at the puncture and not containing other punctures). Then we cut out the disks $|z| \leq r$ and $|w| \leq r$ for some $r=1-\epsilon$ at sewn punctures and identify the annuli $r<|z|<1 / r$ and $r<|w|<1 / r$ via $w=e^{i t} / z$, with $t$ running over the interval $[0,2 \pi]$, and thereby increasing the degree of the chain $\sigma_{i}$. This gives a new chain $S^{1} \times \Delta^{k} \rightarrow \mathcal{M}_{g+1}(n)$, resulting in a new cycle $\Delta(Z)$. Moreover, the map $\Delta$ is well-defined on $H_{\bullet}\left(\mathcal{M}_{g}(n+2)\right)$, i.e., it is independent of the choice of holomorphic disks involved in twist-gluing (see [45, 7]).

2. Similarly, for $n_{1}, n_{2} \geq 0$, define the antibracket

$$
\begin{aligned}
\{-,-\}: H_{k_{1}}\left(\mathcal{M}_{g_{1}}\left(n_{1}+1\right)\right) \otimes H_{k_{2}} & \left(\mathcal{M}_{g_{2}}\left(n_{2}+1\right)\right) \\
& \rightarrow H_{k_{1}+k_{2}+1}\left(\mathcal{M}_{g_{1}+g_{2}}\left(n_{1}+n_{2}\right)\right)
\end{aligned}
$$

as follows. If $Z_{1} \in H_{k_{1}}\left(\mathcal{M}_{g_{1}}\left(n_{1}+1\right)\right)$ and $Z_{2} \in H_{k_{2}}\left(\mathcal{M}_{g_{2}}\left(n_{2}+1\right)\right)$ are homology classes represented by singular cycles $Z_{1}=\sum_{i=1}^{m} c_{i} \sigma_{i}$ for $\sigma_{i}: \Delta^{k_{1}} \rightarrow \mathcal{M}_{g_{1}}\left(n_{1}+1\right)$ and $Z_{2}=\sum_{j=1}^{m} d_{j} \tau_{j}$ for $\tau_{j}: \Delta^{k_{2}} \rightarrow \mathcal{M}_{g_{2}}\left(n_{2}+1\right)$, then set

$$
\left.\left\{Z_{1}, Z_{2}\right\}:=\sum_{i, j} c_{i} d_{j} \sum_{\beta, \gamma} \text { (twist-gluing of } \sigma_{i} \text { with } \tau_{j} \text { at punctures } \beta, \gamma\right),
$$

where $\beta$ runs over the punctures of the surface in $\mathcal{M}_{g_{1}}\left(n_{1}+1\right)$ and $\gamma$ runs over the punctures of the surface in $\mathcal{M}_{g_{2}}\left(n_{2}+1\right)$. This gives a new chain $S^{1} \times \Delta^{k_{1}} \times \Delta^{k_{2}} \rightarrow \mathcal{M}_{g_{1}+g_{2}}\left(n_{1}+n_{2}\right)$, resulting in a new cycle $\left\{Z_{1}, Z_{2}\right\} \in$ $H_{k_{1}+k_{2}+1}\left(\mathcal{M}_{g_{1}+g_{2}}\left(n_{1}+n_{2}\right)\right)$, independent of the choices made along the way (see ibid.).

The reason why these homology operations are introduced is to set up a quantum master equation, actually, at the chain, rather than homology level:

$$
d S+\hbar \Delta S+\frac{1}{2}\{S, S\}=0
$$

on a formal power series $S=\sum_{\substack{g, n \geq 0 \\ 2 g-2+n>0}} S_{g, n} \lambda^{2 g-2+n} \hbar^{g}$ with coefficients $S_{g, n}$ being chains in $C_{6 g-6+2 n}\left(\mathcal{M}_{g}(n)\right)$ and $d$ denoting chain boundary. 
However, for the above equation to be sensible, degree considerations suggest to change grading on chains and homology to grading by codimension, as well as assume that the formal variables $\lambda$ and $\hbar$ have degree zero. This change of grading may be regarded as the application of canonical Poincaré-Lefschetz duality

$$
H_{k}\left(\mathcal{M}_{g}(n)\right)=H_{c}^{6 g-6+2 n-k}\left(\mathcal{M}_{g}(n)\right),
$$

where $H_{c}^{\bullet}$ denotes cohomology with compact support. Thus, under PoincaréLefschetz duality, the BV operator (3) and antibracket (4) turn into

$$
\Delta: H_{c}^{k}\left(\mathcal{M}_{g}(n+2)\right) \rightarrow H_{c}^{k+1}\left(\mathcal{M}_{g+1}(n)\right)
$$

and

$$
\begin{aligned}
\{-,-\}: H_{c}^{k_{1}}\left(\mathcal{M}_{g_{1}}\left(n_{1}+1\right)\right) \otimes H_{c}^{k_{2}} & \left(\mathcal{M}_{g_{2}}\left(n_{2}+1\right)\right) \\
& \rightarrow H_{c}^{k_{1}+k_{2}+1}\left(\mathcal{M}_{g_{1}+g_{2}}\left(n_{1}+n_{2}\right)\right),
\end{aligned}
$$

respectively. We hope that, given that Poincaré-Lefschetz duality identifies these operations (5) and (6) with (3) and (4), respectively, our use of the same notation, $\Delta$ and $\{-,-\}$, will not create serious confusion.

From [2, 45] we know that these two operations define the structure of a dg-Lie algebra with a differential $\Delta$ of degree 1 on

$$
\mathfrak{g}:=\bigoplus_{\substack{g, n \geq 0 \\ 2 g-2+n>0}} H_{c}^{\bullet}\left(\mathcal{M}_{g}(n)\right)[1],
$$

where $V[1]$ denotes the desuspension of a graded vector space $V: V[1]^{k}:=$ $V^{k+1}$.

Remark 1.1. The graded symmetric algebra $S(\mathfrak{g}[-1])$ on the suspension of a dg-Lie algebra $\mathfrak{g}$ is known to carry the structure of a $\mathrm{BV}$-algebra, see [42, Example 2.5], which uses a slightly different grading convention: $\operatorname{deg} \Delta=$ -1 and $\operatorname{deg} \hbar=2$. In the case of our dg-Lie algebra $\mathfrak{g}$ as in (7), the graded symmetric algebra $S(\mathfrak{g}[-1])$ is isomorphic to the (co)homology $H_{c}^{\bullet}(\mathcal{M})$ of Zwiebach's dg-BV-algebra $C_{c}^{\bullet}(\mathcal{M})$ of compactly supported chains in the moduli space $\mathcal{M}$ of not necessarily connected, closed Riemann surfaces with unlabeled punctures. 
1.2. Construction via the real Deligne-Mumford compactification. Let $\underline{\mathcal{M}}_{g}(n)$ be the real Deligne-Mumford compactification of $\mathcal{M}_{g}(n)$ to an orbifold with corners (see [24]). It is known that $\underline{\mathcal{M}}_{g}(n)$ is homotopy equivalent to $\mathcal{M}_{g}(n)$ and $H_{\bullet}\left(\underline{\mathcal{M}}_{g}(n)\right) \cong H_{\bullet}\left(\mathcal{M}_{g}(n)\right)$. In this construction, $\Delta$ uses twist-attaching (in which there are $S^{1}$ ways of attaching a pair of punctures on a Riemann surface $\Sigma \in \underline{\mathcal{M}}_{g}(n)$, or $S^{1}$ worth of choices of real rays in the tensor product (over $\mathbb{C}$ ) of the tangent spaces to $\Sigma$ at these two punctures). The antibracket $\{-,-\}$ uses twist-attaching in a similar way.

\subsection{Construction via the Deligne-Mumford compactification.}

1.3.1. The "topological" spectral sequence. Let $\overline{\mathcal{M}}_{g}(n)$ denote the DeligneMumford (DM) compactification of $\mathcal{M}_{g}(n)$ and $\overline{\mathcal{M}}_{g}(n)^{(p)}$ be the moduli space of Riemann surfaces with at least $p$ double points (also known as nodes). Then we have a decreasing filtration

$$
\cdots \subset \overline{\mathcal{M}}_{g}(n)^{(p+1)} \subset \overline{\mathcal{M}}_{g}(n)^{(p)} \cdots \subset \overline{\mathcal{M}}_{g}(n)^{(0)}=\overline{\mathcal{M}}_{g}(n) .
$$

It generates a spectral sequence satisfying

$$
E_{p, q}^{1}=H_{p+q}\left(\overline{\mathcal{M}}_{g}(n)^{(-p)}, \overline{\mathcal{M}}_{g}(n)^{(-p+1)}\right) \Rightarrow H_{\bullet}\left(\overline{\mathcal{M}}_{g}(n)\right)
$$

with $E_{p, q}^{1}=0$ unless $q \geq-p \geq 0$ and a differential

$$
d_{p, q}^{1}: H_{p+q}\left(\overline{\mathcal{M}}_{g}(n)^{(-p)}, \overline{\mathcal{M}}_{g}(n)^{(-p+1)}\right) \rightarrow H_{p+q-1}\left(\overline{\mathcal{M}}_{g}(n)^{(-p+1)}, \overline{\mathcal{M}}_{g}(n)^{(-p+2)}\right),
$$

given by the boundary map in the long exact sequence of the triple $\left(\overline{\mathcal{M}}_{g}(n)^{(-p)}, \overline{\mathcal{M}}_{g}(n)^{(-p+1)}, \overline{\mathcal{M}}_{g}(n)^{(-p+2)}\right)$. We will also utilize a coboundary map, the linear dual

$$
\left(d_{p, q}^{1}\right)^{*}: H_{c}^{p+q-1}\left(\overline{\mathcal{M}}_{g}(n)^{(-p+1)} \backslash \overline{\mathcal{M}}_{g}(n)^{(-p+2)}\right) \rightarrow H_{c}^{p+q}\left(\overline{\mathcal{M}}_{g}(n)^{(-p)} \backslash \overline{\mathcal{M}}_{g}(n)^{(-p+1)}\right)
$$

of $d_{p, q}^{1}$ later on.

After applying Poincaré-Lefschetz duality and a linear change of variables $p, q$ to the spectral sequence (8), we get a cohomological spectral sequence with

$$
E_{1}^{p, q}=H^{q-p}\left(\overline{\mathcal{M}}_{g}(n)^{(p)} \backslash \overline{\mathcal{M}}_{g}(n)^{(p+1)}\right) \Rightarrow H^{\bullet}\left(\overline{\mathcal{M}}_{g}(n)\right)
$$

with $q \geq p \geq 0$ and a differential

$$
d_{1}^{p, q}: H^{q-p}\left(\overline{\mathcal{M}}_{g}(n)^{(p)} \backslash \overline{\mathcal{M}}_{g}(n)^{(p+1)}\right) \rightarrow H^{q-p-1}\left(\overline{\mathcal{M}}_{g}(n)^{(p+1)} \backslash \overline{\mathcal{M}}_{g}(n)^{(p+2)}\right) .
$$


This is the Poincaré residue map (see [8, 11, 25]), which might be thought of as a Gysin homomorphism, integration along the boundary of the tubular neighborhood of $\overline{\mathcal{M}}_{g}(n)^{(p+1)}$ inside $\overline{\mathcal{M}}_{g}(n)^{(p)}$. The linear dual is

$$
\left(d_{1}^{p, q}\right)^{*}: H_{q-p-1}\left(\overline{\mathcal{M}}_{g}(n)^{(p+1)} \backslash \overline{\mathcal{M}}_{g}(n)^{(p+2)}\right) \rightarrow H_{q-p}\left(\overline{\mathcal{M}}_{g}(n)^{(p)} \backslash \overline{\mathcal{M}}_{g}(n)^{(p+1)}\right)
$$

may also be thought of as a Gysin homomorphism in homology. We will now demonstrate that the BV operator (3) and antibracket (4) are essentially these Gysin homomorphisms $\left(d_{1}^{p, q}\right)^{*}$, see (11), for $p=0$. This will imply that the corresponding operators (5) and (6) on the compactly supported cohomology are essentially the coboundary homomorphisms $\left(d_{p, q}^{1}\right)^{*}$, see (9), for $p=0$.

\subsubsection{Identification of the BV operator. Let}

$$
\begin{aligned}
\alpha_{p}: H_{\bullet}\left(\overline{\mathcal{M}}_{g}(n+2)^{(p)} \backslash \overline{\mathcal{M}}_{g}(\right. & \left.+2)^{(p+1)}\right) \\
& \rightarrow H_{\bullet}\left(\overline{\mathcal{M}}_{g+1}(n)^{(p+1)} \backslash \overline{\mathcal{M}}_{g+1}(n)^{(p+2)}\right)
\end{aligned}
$$

be the composition $\alpha_{p}:=\pi_{*} \rho$, where $\pi_{*}$ is the pushforward of the map $\pi$ attaching the last two punctures and $\rho^{!}$is the transfer map for $\rho$ from the following diagram of étale morphisms:

$$
\begin{aligned}
&\left(\overline{\mathcal{M}}_{g, n+2}^{(p)} \backslash \overline{\mathcal{M}}_{g, n+2}^{(p+1)}\right) / S_{n} \times S_{2} \stackrel{\pi}{\longrightarrow} \overline{\mathcal{M}}_{g+1}(n)^{(p+1)} \backslash \overline{\mathcal{M}}_{g+1}(n)^{(p+2)} \\
& \\
& \overline{\mathcal{M}}_{g}(n+2)^{(p)} \backslash \backslash \overline{\mathcal{M}}_{g}(n+2)^{(p+1)},
\end{aligned}
$$

in which $\overline{\mathcal{M}}_{g, n+2}^{(p)}$ is the moduli space of Riemann surfaces with $n+2$ labeled punctures and at least $p$ nodes, $S_{n}$ permutes the first $n$ punctures in $\mathcal{M}_{g, n+2}$, whereas $S_{2}$ permutes the last two punctures, and $\rho$ forgets the division of the punctures into two groups: the first $n$ ones and the last two.

Let us consider the particular case $p=0$, where $\overline{\mathcal{M}}_{g}(n+2)^{(0)} \backslash \overline{\mathcal{M}}_{g}(n+$ $2)^{(1)}=\mathcal{M}_{g}(n+2)$ and $\overline{\mathcal{M}}_{g+1}(n)^{(1)} \backslash \overline{\mathcal{M}}_{g+1}(n)^{(2)}$ is the moduli space of Riemann surfaces with exactly one node. Then we have

$$
\alpha_{0}: H_{\bullet}\left(\mathcal{M}_{g}(n+2)\right) \rightarrow H_{\bullet}\left(\overline{\mathcal{M}}_{g+1}(n)^{(1)} \backslash \overline{\mathcal{M}}_{g+1}(n)^{(2)}\right),
$$


where $\alpha_{0}=\pi_{*} \rho^{!}$for the diagram

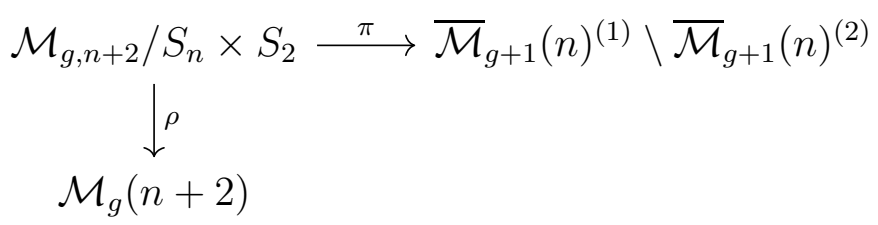

of étale morphisms.

Theorem 1.2. (1) The BV operator $\Delta$ as in Eq. (3) is equal to the composition $\left(d_{1}^{0, \bullet}\right)^{*} \alpha_{0}$ below:

$$
\begin{aligned}
& H_{\bullet}\left(\mathcal{M}_{g}(n+2)\right) \stackrel{\alpha_{0}}{\longrightarrow} H_{\bullet}\left(\overline{\mathcal{M}}_{g+1}(n)^{(1)} \backslash \overline{\mathcal{M}}_{g+1}(n)^{(2)}\right) \\
& \stackrel{\left(d_{1}^{0, \bullet}\right)^{*}}{\longrightarrow} H_{\bullet+1}\left(\mathcal{M}_{g+1}(n)\right) .
\end{aligned}
$$

(2) The BV operator $\Delta$ as in Eq. (5) is equal to the composition $\left(d_{0, \bullet}^{1}\right)^{*} \alpha_{0}$ below:

$$
\begin{aligned}
& H_{c}^{\bullet}\left(\mathcal{M}_{g}(n+2)\right) \stackrel{\alpha_{0}}{\longrightarrow} H_{c}^{\bullet}\left(\overline{\mathcal{M}}_{g+1}(n)^{(1)} \backslash \overline{\mathcal{M}}_{g+1}(n)^{(2)}\right) \\
& \stackrel{\left(d_{0, \bullet}^{1}\right)^{*}}{\longrightarrow} H_{c}^{\bullet+1}\left(\mathcal{M}_{g+1}(n)\right),
\end{aligned}
$$

where $\alpha_{0}=\pi_{*} \rho$ ! is the morphism induced by the étale morphisms $\pi$ and $\rho$ from (12) on cohomology with compact support.

Proof. Claims (1) and (2) are equivalent by Poincaré-Lefschetz duality. To prove claim (1), let us trace what happens under the maps $\rho^{!}$and $\pi_{*}$ mapking up the map $\alpha_{0}$, as well as under the map $\left(d_{1}^{0, \bullet}\right)^{*}$. Given a singular chain of smooth Riemann surfaces of genus $g$ with $n+2$ unlabeled punctures, the transfer map $\rho^{!}$sums up all possible ways of picking a pair of punctures, whereas the map $\pi^{*}$ attaches these two punctures to form a node and thereby places this chain within the part $\overline{\mathcal{M}}_{g+1}(n)^{(1)} \backslash \overline{\mathcal{M}}_{g+1}(n)^{(2)}$ of the DeligneMumford compactification $\overline{\mathcal{M}}_{g+1}(n)$ which corresponds to stable curves with exactly one node. This is exactly what the BV operator $\Delta$ does, except that twist-gluing at the chosen pair of punctures is replaced so far with attaching. Now, the map $\left(d_{1}^{0, \bullet}\right)^{*}$ in homology linear dual to the Poincaré residue map $d_{1}^{0, \bullet}$ in cohomology is the umkehr map, which associates to a cycle in $\overline{\mathcal{M}}_{g+1}(n)^{(1)} \backslash \overline{\mathcal{M}}_{g+1}(n)^{(2)}$ its pre-image in the boundary of the 
tubular neighborhood of $\overline{\mathcal{M}}_{g+1}(n)^{(1)} \backslash \overline{\mathcal{M}}_{g+1}(n)^{(2)}$ inside $\overline{\mathcal{M}}_{g+1}(n)$. The tubular neighborhood forms an $S^{1}$-bundle over $\overline{\mathcal{M}}_{g+1}(n)^{(1)} \backslash \overline{\mathcal{M}}_{g+1}(n)^{(2)}$ and may locally be built out of Riemann surfaces obtained by twist-gluing at the node of a surface in $\overline{\mathcal{M}}_{g+1}(n)^{(1)} \backslash \overline{\mathcal{M}}_{g+1}(n)^{(2)}$. Thus, composing the map $\left(d_{1}^{0, \bullet}\right)^{*}$ sends the homology class of stable curves with one node to the homology class obtained by twist-gluing of stable curves at the node. This reconciles the composition $\left(d_{1}^{0, \bullet}\right)^{*} \alpha_{0}$ with $\Delta$.

1.3.3. Identification of the antibracket. Given that the antibracket has the same nature as the BV operator and is, namely, the derived bracket for the BV operator $\Delta$ on $S(\mathfrak{g}[-1]) \cong H_{\bullet}(\mathcal{M})$, see Remark 1.1, it is not surprising that there is a similar identification of the antibracket via the Gysin homomorphisms, coming from the topological spectral sequences.

A diagram analogous to diagram (12), which defines the homomorphism $\alpha_{0}$, is the following pair of étale morphisms:

$$
\begin{aligned}
\mathcal{M}_{g_{1}, n_{1}+1} / S_{n_{1}} & \times \mathcal{M}_{g_{2}, n_{2}+1} / S_{n_{2}} \stackrel{\pi}{\longrightarrow} \overline{\mathcal{M}}_{g_{1}+g_{2}}\left(n_{1}+n_{2}\right)^{(1)} \backslash \overline{\mathcal{M}}_{g_{1}+g_{2}}\left(n_{1}+n_{2}\right)^{(2)} \\
& \stackrel{\downarrow \rho}{ } \\
\mathcal{M}_{g_{1}}\left(n_{1}+1\right) & \times \mathcal{M}_{g_{2}}\left(n_{2}+1\right) .
\end{aligned}
$$

Here $S_{n_{i}}$ permutes the first $n_{i}$ punctures in $\mathcal{M}_{g_{i}, n_{i}+1}, i=1,2$, and $\pi$ attaches the last, $n_{1}+1$ st puncture on the Riemann surface representing a point in $\mathcal{M}_{g_{1}, n_{1}+1} / S_{n_{1}}$ to the last, $n_{2}+1$ st puncture on the Riemann surface representing a point in $\mathcal{M}_{g_{2}, n_{2}+1} / S_{n_{2}}$. A diagram analogous to the one defining $\alpha_{p}$ may be written down similarly, but we are skipping it for the sake of simplicity. Define a map

$$
\begin{aligned}
\beta_{0}: H_{\bullet}\left(\mathcal{M}_{g_{1}}\left(n_{1}+1\right)\right) & \otimes H_{\bullet}\left(\mathcal{M}_{g_{2}}\left(n_{2}+1\right)\right) \\
& \rightarrow H_{\bullet}\left(\overline{\mathcal{M}}_{g_{1}+g_{2}}\left(n_{1}+n_{2}\right)^{(1)} \backslash \overline{\mathcal{M}}_{g_{1}+g_{2}}\left(n_{1}+n_{2}\right)^{(2)}\right)
\end{aligned}
$$

as $\beta_{0}=\pi_{*} \rho^{!}$for $\pi$ and $\rho$ from the previous diagram. The following theorem is proven exactly in the same way as Theorem 1.2 . 
Theorem 1.3. (1) The antibracket $\{-,-\}$ as in Eq. (4) is equal to the composition $\left(d_{1}^{0, \bullet}\right)^{*} \beta_{0}$ below:

$$
\begin{aligned}
H_{\bullet}\left(\mathcal{M}_{g_{1}}\left(n_{1}+1\right)\right) \otimes H_{\bullet}\left(\mathcal{M}_{g_{2}}\left(n_{2}+1\right)\right) & \\
\stackrel{\beta_{0}}{\longrightarrow} H_{\bullet}\left(\overline{\mathcal{M}}_{g_{1}+g_{2}}\left(n_{1}+n_{2}\right)^{(1)} \backslash \overline{\mathcal{M}}_{g_{1}+g_{2}}\left(n_{1}+n_{2}\right)^{(2)}\right) & \\
& \stackrel{\left(d_{1}^{0, \bullet}\right)^{*}}{\longrightarrow} H_{\bullet+1}\left(\mathcal{M}_{g_{1}+g_{2}}\left(n_{1}+n_{2}\right)\right) .
\end{aligned}
$$

(2) The antibracket $\{-,-\}$ as in Eq. (6) is equal to the composition $\left(d_{0, \bullet}^{1}\right)^{*} \beta_{0}$ below:

$$
\begin{aligned}
H_{c}^{\bullet}\left(\mathcal{M}_{g_{1}}\left(n_{1}+1\right)\right) \otimes H_{c}^{\bullet}\left(\mathcal{M}_{g_{2}}\left(n_{2}+1\right)\right) & \\
\stackrel{\beta_{0}}{\longrightarrow} H_{c}^{\bullet}\left(\overline{\mathcal{M}}_{g_{1}+g_{2}}\left(n_{1}+n_{2}\right)^{(1)} \backslash \overline{\mathcal{M}}_{g_{1}+g_{2}}\left(n_{1}+n_{2}\right)^{(2)}\right) & \\
& \stackrel{\left(d_{0, \bullet}^{1}\right)^{*}}{\longrightarrow} H_{c}^{\bullet+1}\left(\mathcal{M}_{g_{1}+g_{2}}\left(n_{1}+n_{2}\right)\right),
\end{aligned}
$$

where $\beta_{0}=\pi_{*} \rho$ ! is the morphism induced by the étale morphisms $\pi$ and $\rho$ from (13) on cohomology with compact support.

\section{VANISHING RESULTS FOR THE BV OPERATOR AND ANTIBRACKET}

2.1. The genus $g=0$ case. Let us consider the case $g=0$ first, as the BV operator and antibracket vanish on the moduli space of genus-zero Riemann surfaces for the trivial reason of it being rationally acyclic.

\section{Theorem 2.1.}

$$
H^{k}\left(\mathcal{M}_{0}(n)\right)= \begin{cases}\mathbb{Q}, & \text { if } k=0 \\ 0, & \text { otherwise }\end{cases}
$$

This theorem follows from Arnold's computation [1, 4] of the rational cohomology of the braid group, as observed by Looijenga [27]. See also a different argument of Westerland [43]. We also need the following classical result.

Theorem 2.2 (Mumford [31]). $H^{1}\left(\mathcal{M}_{1, n}\right)=0$.

These results imply the desired vanishing. 
Corollary 2.3. 1 . The BV operator

$$
H_{k}\left(\mathcal{M}_{0}(n+2)\right) \stackrel{\Delta}{\rightarrow} H_{k+1}\left(\mathcal{M}_{1}(n)\right)
$$

vanishes for all $k \geq 0, n \geq 1$. In cohomology with compact support,

$$
H_{c}^{l}\left(\mathcal{M}_{0}(n+2)\right) \stackrel{\Delta}{\rightarrow} H_{c}^{l+1}\left(\mathcal{M}_{1}(n)\right)
$$

vanishes for all $l \geq 0, n \geq 1$. 2. The antibracket

$$
H_{k_{1}}\left(\mathcal{M}_{0}\left(n_{1}+1\right)\right) \otimes H_{k_{2}}\left(\mathcal{M}_{0}\left(n_{2}+1\right)\right) \stackrel{\{-,-\}}{\longrightarrow} H_{k_{1}+k_{2}+1}\left(\mathcal{M}_{0}\left(n_{1}+n_{2}\right)\right)
$$

vanishes for $k_{1}, k_{2} \geq 0, n_{1}, n_{2} \geq 1$. In cohomology with compact support, the antibracket

$$
H_{c}^{l_{1}}\left(\mathcal{M}_{0}\left(n_{1}+1\right)\right) \otimes H_{c}^{l_{2}}\left(\mathcal{M}_{0}\left(n_{2}+1\right)\right) \stackrel{\{-,-\}}{\longrightarrow} H_{c}^{l_{1}+l_{2}+1}\left(\mathcal{M}_{0}\left(n_{1}+n_{2}\right)\right)
$$

vanishes when $l_{1}, l_{2} \geq 0, n_{1}, n_{2} \geq 1$.

2.2. The genus $g>0$ case. From [14] we have the following bounds on homology:

$$
H_{k}\left(\mathcal{M}_{g, n}\right)=0 \quad \text { for } \quad\left\{\begin{array}{l}
g=0, k>n-3 \\
n=0, k>4 g-5 \\
n>0, g>0, k>4 g-4+n
\end{array}\right.
$$

Equivalently,

$$
H_{c}^{l}\left(\mathcal{M}_{g, n}\right)=0 \quad \text { for } \quad\left\{\begin{array}{l}
g=0, l<n-3, \\
n=0, l<2 g-1, \\
n>0, g>0, l<2 g-2+n
\end{array}\right.
$$

These above bounds imply the following. For the BV operator

$$
H_{k}\left(\mathcal{M}_{g}(n+2)\right) \stackrel{\Delta}{\rightarrow} H_{k+1}\left(\mathcal{M}_{g+1}(n)\right)
$$

as in Equation (3), the left-hand side vanishes for $k>4 g-2+n, n \geq 0$, while the right-hand side is zero for $k>4 g-1+n$, if $n>0$, and $k>4 g-2$, if $n=0$. On the other hand, for the antibracket

$$
H_{k_{1}}\left(\mathcal{M}_{g_{1}}\left(n_{1}+1\right)\right) \otimes H_{k_{2}}\left(\mathcal{M}_{g_{2}}\left(n_{2}+1\right)\right) \stackrel{\{-,-\}}{\longrightarrow} H_{k_{1}+k_{2}+1}\left(\mathcal{M}_{g_{1}+g_{2}}\left(n_{1}+n_{2}\right)\right)
$$


as in Equation (4), the left-hand side is zero for $k_{1}>4 g_{1}-3+n_{1}$ or $k_{2}>4 g_{2}-3+n_{2}$, but the right-hand side vanishes for $k_{1}+k_{2}>4 g_{1}+$ $4 g_{2}-5+n_{1}+n_{2}$, if $n_{1}+n_{2}>0$, and $k_{1}+k_{2}>4 g_{1}+4 g_{2}-6$, if $n_{1}+n_{2}=0$. Therefore, we have

Theorem 2.4. 1. The BV operator

$$
H_{k}\left(\mathcal{M}_{g}(n+2)\right) \stackrel{\Delta}{\rightarrow} H_{k+1}\left(\mathcal{M}_{g+1}(n)\right)
$$

vanishes for $k>4 g-2+n, g>0, n \geq 0$. In cohomology with compact support,

$$
H_{c}^{l}\left(\mathcal{M}_{g}(n+2)\right) \stackrel{\Delta}{\rightarrow} H_{c}^{l+1}\left(\mathcal{M}_{g+1}(n)\right)
$$

vanishes for $l<2 g+n, g>0, n \geq 0$. In particular, $\Delta=0$ on compactly supported cohomology as $g \rightarrow \infty$ or $n \rightarrow \infty$ and l being fixed, i.e., stably

2. The antibracket

$H_{k_{1}}\left(\mathcal{M}_{g_{1}}\left(n_{1}+1\right)\right) \otimes H_{k_{2}}\left(\mathcal{M}_{g_{2}}\left(n_{2}+1\right)\right) \stackrel{\{-,-\}}{\longrightarrow} H_{k_{1}+k_{2}+1}\left(\mathcal{M}_{g_{1}+g_{2}}\left(n_{1}+n_{2}\right)\right)$ vanishes for $k_{1}>4 g_{1}-3+n_{1}$ or $k_{2}>4 g_{2}-3+n_{2}, g_{1}, g_{2}>0$ and $n_{1}, n_{2} \geq 0$. In cohomology with compact support, the antibracket

$$
H_{c}^{l_{1}}\left(\mathcal{M}_{g_{1}}\left(n_{1}+1\right)\right) \otimes H_{c}^{l_{2}}\left(\mathcal{M}_{g_{2}}\left(n_{2}+1\right)\right) \stackrel{\{-,-\}}{\longrightarrow} H_{c}^{l_{1}+l_{2}+1}\left(\mathcal{M}_{g_{1}+g_{2}}\left(n_{1}+n_{2}\right)\right)
$$

vanishes when $l_{1}<2 g_{1}-1+n_{1}$ or $l_{2}<2 g_{2}-1+n_{2}, g_{1}, g_{2}>0$ and $n_{1}, n_{2} \geq 0$.

Likewise, homological stability implies the vanishing of the BV operator and antibracket within the stable range $k<\frac{2}{3}(g-1)$. Indeed, Harer's stability theorem [13], as improved by Ivanov [17, 18, 19], Harer himself [15], Boldsen [3], and Randal-Williams [34]:

$$
H^{k}\left(\mathcal{M}_{g}\right) \cong H^{k}\left(\mathcal{M}_{g+1}\right) \quad \text { for } k<\frac{2}{3}(g-1),
$$

combined with Madsen and Weiss's proof [29] of Mumford's conjecture, stating that

$$
\mathbb{Q}\left[\kappa_{1}, \kappa_{2}, \ldots\right] \rightarrow H^{\bullet}\left(\mathcal{M}_{g}\right),
$$

where $\kappa_{i} \in H^{2 i}\left(\mathcal{M}_{g}\right)$ is the $i$ th "tautological" $\kappa$ class, $i=1,2, \ldots$, is an isomorphism in degree $\leq \frac{2}{3}(g-1)$, and Looijenga's relation [28] with the 
case of pointed Riemann surfaces, which asserts that

$$
H^{\bullet}\left(\mathcal{M}_{g}\right)\left[\psi_{1}, \psi_{2}, \ldots, \psi_{n}\right] \rightarrow H^{\bullet}\left(\mathcal{M}_{g, n}\right),
$$

where $\psi_{i} \in H^{2}\left(M_{g}\right)$ is the $i$ th "tautological" $\psi$ class, $i=1, \ldots, n$, is an isomorphism in degree $\leq \frac{2}{3}(g-1)$, implies that

$$
\mathbb{Q}\left[\psi_{1}, \ldots, \psi_{n}, \kappa_{1}, \kappa_{2}, \ldots\right] \rightarrow H^{\bullet}\left(\mathcal{M}_{g, n}\right)
$$

is an isomorphism in degree $\leq \frac{2}{3}(g-1)$. This, in particular, forces the rational cohomology $H^{\bullet}\left(\mathcal{M}_{g, n}\right)$ in the stable range to be concentrated in even degrees. Taking invariants of the $S_{n}$-action on cohomology does not affect these statements. Now, given that the BV operator and antibracket have degree 1 , we obtain the following vanishing result.

Theorem 2.5. 1. The BV operator

$$
H_{k}\left(\mathcal{M}_{g}(n+2)\right) \stackrel{\Delta}{\rightarrow} H_{k+1}\left(\mathcal{M}_{g+1}(n)\right)
$$

vanishes for $k \leq \frac{2}{3}(g-1), g>0, n \geq 0$. In particular, $\Delta=0$ on homology as $g \rightarrow \infty$ or $n \rightarrow \infty$ and $k$ being fixed, i.e., stably. In cohomology with compact support,

$$
H_{c}^{l}\left(\mathcal{M}_{g}(n+2)\right) \stackrel{\Delta}{\rightarrow} H_{c}^{l+1}\left(\mathcal{M}_{g+1}(n)\right)
$$

vanishes for $l \geq \frac{16}{3} g-\frac{4}{3}+2 n, g>0, n \geq 0$.

\section{The antibracket}

$H_{k_{1}}\left(\mathcal{M}_{g_{1}}\left(n_{1}+1\right)\right) \otimes H_{k_{2}}\left(\mathcal{M}_{g_{2}}\left(n_{2}+1\right)\right) \stackrel{\{-,-\}}{\longrightarrow} H_{k_{1}+k_{2}+1}\left(\mathcal{M}_{g_{1}+g_{2}}\left(n_{1}+n_{2}\right)\right)$

vanishes for $k_{1} \leq \frac{2}{3}\left(g_{1}-1\right), k_{2} \leq \frac{2}{3}\left(g_{2}-1\right), k_{1}+k_{2} \leq \frac{2}{3}\left(g_{1}+g_{2}\right)-\frac{5}{3}$, $g_{1}, g_{2}>0$, and $n_{1}, n_{2} \geq 0$. In cohomology with compact support, the antibracket

$$
H_{c}^{l_{1}}\left(\mathcal{M}_{g_{1}}\left(n_{1}+1\right)\right) \otimes H_{c}^{l_{2}}\left(\mathcal{M}_{g_{2}}\left(n_{2}+1\right)\right) \stackrel{\{-,-\}}{\longrightarrow} H_{c}^{l_{1}+l_{2}+1}\left(\mathcal{M}_{g_{1}+g_{2}}\left(n_{1}+n_{2}\right)\right)
$$

vanishes when $l_{1} \geq \frac{16}{3} g_{1}-\frac{10}{3}+2 n_{1}, l_{2} \geq \frac{16}{3} g_{2}-\frac{10}{3}+2 n_{2}, l_{1}+l_{2} \geq$ $\frac{16}{3}\left(g_{1}+g_{2}\right)-\frac{19}{3}+2\left(n_{1}+n_{2}\right), g_{1}, g_{2}>0$, and $n_{1}, n_{2} \geq 0$.

2.3. A nonvanishing example. Given a vanishing range of the BV operator and antibracket on rational homology, one may wonder if they vanish identically. Concrete computations with moduli spaces are quite hard in 
general, and we do not have an example of nonvanishing of the antibracket. Here we present an example of nonvanishing of the BV operator. First off, let us analyze the differential in the spectral sequence (10).

Proposition 2.6. The differential $d_{1}^{0,6}: H^{6}\left(\mathcal{M}_{3}\right) \rightarrow H^{5}\left(\overline{\mathcal{M}}_{3}^{(1)} \backslash \overline{\mathcal{M}}_{3}^{(2)}\right)$ is nontrivial. Moreover, so is its projection to $H^{5}\left(\mathcal{M}_{2}(2)\right)$.

Proof. It suffices to prove the second statement. By Looijenga's computation [27] of the rational cohomology of $\mathcal{M}_{3}$, its Poincaré-Serre polynomial, in which the coefficient by $t^{k} u^{l}$ is the dimension of the subquotient $H^{k}\left(\mathcal{M}_{3}\right)$ of weight $l$, is equal to $1+t^{2} u^{2}+t^{6} u^{12}$. This implies that $H^{6}\left(\mathcal{M}_{3}\right) \cong \mathbb{Q}$ of weight 12 . Since the weight is not equal to the cohomology degree, the corresponding element in $E_{1}^{0,6}$ will not survive to $H^{6}\left(\overline{\mathcal{M}}_{3}\right)$ in the limit $E_{\infty}^{0,6}$. If all the differentials $d_{r}^{0,6}, r \geq 1$, were zero on $H^{6}\left(\mathcal{M}_{3}\right)$, then $H^{6}\left(\mathcal{M}_{3}\right)$ would contribute nontrivially to $E_{\infty}^{0,6} \subset H^{6}\left(\overline{\mathcal{M}}_{3}\right)$ and thereby have weight 6 , which would be contradiction. The plan is to show that all the higher differentials $d_{r}^{0,6}, r \geq 2$, vanish. This would force $d_{1}^{0,6}$ to be nontrivial.

Now, looking at different components of the boundary $\partial \mathcal{M}_{3}$, we find that they all have a trivial cohomology group $H^{5}$, except possibly $\mathcal{M}_{2}(2)$. Indeed, $\overline{\mathcal{M}}_{3}^{(1)} \backslash \overline{\mathcal{M}}_{3}^{(2)}=\mathcal{M}_{2}(2) \coprod \mathcal{M}_{2,1} \times \mathcal{M}_{1,1}$. The space $\mathcal{M}_{1,1}$ of elliptic curves is known to be isomorphic to the affine line $\mathbb{C}$, whereas the following argument, borrowed from Dan Petersen [33], shows that $\mathcal{M}_{2,1}$ has the rational homology of $\mathbb{C P}^{1}$. Indeed, consider the Leray-Serre spectral sequence for the forgetful map $\pi: \mathcal{M}_{2,1} \rightarrow \mathcal{M}_{2}$. The base $\mathcal{M}_{2}$ is isomorphic to $\mathcal{M}_{0}(6)$, because every curve of genus 2 is hyperelliptic. We know from Theorem 2.1 that it has the cohomology of a singleton. On the other hand, the local systems $R^{0} \pi_{*} \mathbb{Q}$ and $R^{2} \pi_{*} \mathbb{Q}$ are trivial, while $R^{1} \pi_{*} \mathbb{Q}$ has no rational cohomology, as the curve representing a point of $\mathcal{M}_{2}$ has the hyperelliptic involution, which acts on the fiber of $R^{1} \pi_{*} \mathbb{Q}$ by -1 .

We conclude that the projection of $d_{1}^{0,6}: H^{6}\left(\mathcal{M}_{3}\right) \rightarrow H^{5}\left(\overline{\mathcal{M}}_{3}^{(1)} \backslash \overline{\mathcal{M}}_{3}^{(2)}\right)$ onto $H^{5}\left(\mathcal{M}_{2,1} \times \mathcal{M}_{1,1}\right)=0$ must be zero. If we show that the higher differentials $d_{r}^{0,6}, r \geq 2$, vanish, it will imply that the projection of $d_{1}^{0,6}$ : $H^{6}\left(\mathcal{M}_{3}\right) \rightarrow H^{5}\left(\overline{\mathcal{M}}_{3}^{(1)} \backslash \overline{\mathcal{M}}_{3}^{(2)}\right)$ to $H^{5}\left(\mathcal{M}_{2}(2)\right)$ is nontrivial. 
For each $r \geq 2$, the higher differential $d_{r}^{0,6}$ maps $E_{r}^{0,6}$, which is a subspace of $E_{1}^{0,6}$, to $E_{r}^{r, 5+r}$, which is a subquotient of $E_{1}^{r, 5+r}$. We claim that all these terms $E_{1}^{r, 5+r}$ are zero. For $r=2$, the term $E_{1}^{r, 5+r}=E_{1}^{2,7}=H^{5}\left(\overline{\mathcal{M}}_{3}^{(2)} \backslash\right.$ $\overline{\mathcal{M}}_{3}^{(3)}$ ) is the direct sum of the cohomology groups of connected components of $\left.\overline{\mathcal{M}}_{3}^{(2)} \backslash \overline{\mathcal{M}}_{3}^{(3)}\right)$. These components are quotients under finite group actions of the following spaces:

$$
\mathcal{M}_{1,4}, \quad \mathcal{M}_{2,1} \times \mathcal{M}_{0,3}, \quad \mathcal{M}_{1,3} \times \mathcal{M}_{1,1}, \quad \mathcal{M}_{1,2} \times \mathcal{M}_{1,1} \times \mathcal{M}_{1,1}
$$

Looking at the forgetful map $\mathcal{M}_{1,4} \rightarrow \mathcal{M}_{1,1}$, which is topologically a fiber bundle with fiber of the homotopy type of a three-dimensional $\mathrm{CW}$ complex and base having the homotopy type of a point, we see that $H^{5}\left(\mathcal{M}_{1,4}\right)=0$. We have already seen that $\mathcal{M}_{2,1}$ has the rational homology of $\mathbb{C P}^{1}$, and so does $\mathcal{M}_{2,1} \times \mathcal{M}_{0,3}$. Similar to $\mathcal{M}_{1,4}$, the space $\mathcal{M}_{1,3}$ has the homotopy type of a CW complex of dimension two, and so does $\mathcal{M}_{1,3} \times \mathcal{M}_{1,1}$. A similiar argument works for $\mathcal{M}_{1,2} \times \mathcal{M}_{1,1} \times \mathcal{M}_{1,1}$.

Analyzing similarly the groups $E_{1}^{r, 5+r}=H^{5}\left(\overline{\mathcal{M}}_{3}^{(r)} \backslash \overline{\mathcal{M}}_{3}^{(r+1)}\right)$ for $r \geq 3$, we quickly see that all of them vanish for dimensional reasons.

\section{Corollary 2.7. The BV operator}

$$
\Delta: H_{5}\left(\mathcal{M}_{2}(2)\right) \rightarrow H_{6}\left(\mathcal{M}_{3}\right)
$$

does not vanish and is moreover an isomorphism between these one-dimensional vector spaces over $\mathbb{Q}$.

Proof. Working at the dual, cohomology level in the proof of Proposition 2.6, we have seen that $H^{6}\left(\mathcal{M}_{3}\right)$ is one-dimensional. We also have $H^{5}\left(\mathcal{M}_{2}(2)\right)=\mathbb{Q}$, given Tommasi's computation [41, Corollary III.2.2] of the Poincaré-Serre polynomial of $\mathcal{M}_{2}(2)$ as $1+t^{2} u^{2}+t^{5} u^{10}$.

Thus, to prove our claim, we only need to see that $\Delta \neq 0$. By Theorem 1.2, we know that $\Delta=\left(d_{1}^{0,6}\right)^{*} \alpha_{0}$. Recall that $\alpha_{0}=\pi_{*} \rho^{!}$, where $\pi$ and $\rho$ were given in diagram (12). Note that in this particular case, $\rho=\mathrm{id}$ and $\pi$ is the inclusion of $\mathcal{M}_{2}(2)$ as a connected component of $\overline{\mathcal{M}}_{3}^{(1)} \backslash \overline{\mathcal{M}}_{3}^{(2)}$. Thus, $\Delta=\left(d_{1}^{0,6}\right)^{*} \pi_{*}$, which is exactly the linear dual of the projection of $d_{1}^{0,6}$ to $H^{5}\left(\mathcal{M}_{2}(2)\right)$, whose nonvanishing is proven in Proposition 2.6 . 


\section{REFERENCES}

[1] V. I. Arnold, Certain topological invariants of algebraic functions, (Russian) Trudy Moskov. Mat. Obsc. 21 (1970) 27-46; translation in Trans. Moscow Math. Soc. 21 (1970) 30-52.

[2] S. Barannikov, Modular operads and Batalin-Vilkovisky geometry, Int. Math. Res. Not. IMRN (2007), no. 19, Art. ID rnm075, 31.

[3] S. Boldsen, Improved homological stability for the mapping class group with integral or twisted coefficients, Mathematische Zeitschrift 270 (2012), no. 1-2, 297329.

[4] E. Brieskorn, Sur les groupes de tresses, Sminaire Bourbaki 14 (1971-1972) 2144.

[5] K. Cieliebak, K. Fukaya, J. Latschev, Homological algebra related to surfaces with boundary, Preprint, arXiv:1508.02741.

[6] K. Cieliebak, J. Latschev, The role of string topology in symplectic field theory, New perspectives and challenges in symplectic field theory, CRM Proc. Lecture Notes, 49, Amer. Math. Soc., Providence, RI, 2009, pp. 113-146.

[7] K. Costello, The partition function of a topological field theory, Journal of Topology 2 (2009) 779-822.

[8] P. Deligne, Théorie de Hodge : II, Publications Mathématiques de l'I.H.É.S., 40 (1971) 5-57.

[9] M. Doubek, M. Markl, Open-closed modular operads, the Cardy condition and string field theory, J. Noncommut. Geom. 12 (2018), no. 4, 1359-1424.

[10] Y. Eliashberg, A. Givental, H. Hofer, Introduction to symplectic field theory, GAFA 2000 (Tel Aviv, 1999), Geom. Funct. Anal. Special Volume, Part II (2000), 560673.

[11] E. Getzler, Operads and moduli spaces of genus 0 Riemann surfaces, The moduli space of curves, Birkhäuser Boston, 1995, pp. 199-230.

[12] E. Getzler, Topological Recursion Relations in Genus 2, Integrable systems and algebraic geometry (Kobe/Kyoto, 1997), World Sci. Publishing, River Edge, NJ, 1998, pp. 73-106.

[13] J. L. Harer, Stability of the homology of the mapping class groups of orientable surfaces, Ann. of Math. (2), 121(2), 215-249, 1985.

[14] J. L. Harer, The virtual cohomological dimension of the mapping class group of an orientable surface, Invent. Math. 84 (1986) 157-176.

[15] J. L. Harer, Improved stability for the homology of the mapping class groups of surfaces, Preprint, 1993.

[16] E. Harrelson, A. Voronov, J. Zuniga, Open-closed moduli spaces and related algebraic structures, Lett. Math. Phys. 94 (2010), no. 1, 1-26. 
[17] N. V. Ivanov, Complexes of curves and the Teichmüller modular group. (Russian) Uspekhi Mat. Nauk, 42(3), 49-91, 1987; translation in Russian Math. Surveys, 42(3), 55-107, 1987.

[18] N. V. Ivanov, Stabilization of the homology of Teichmüller modular groups. (Russian) Algebra i Analiz, 1(3), 110-126, 1989; translation in Leningrad Math. J., 1(3), 675-691, 1990.

[19] N. V. Ivanov, On the homology stability for Teichmüller modular groups: closed surfaces and twisted coefficients, Mapping class groups and moduli spaces of Riemann surfaces (Göttingen, 1991/Seattle, WA, 1991), Contemp. Math., 150, Amer. Math. Soc., Providence, RI, 1993, pp. 149-194.

[20] B. Jurčo, K. Münster, Type II superstring field theory: geometric approach and operadic description, J. High Energy Phys. 2013, no. 4, 126, front matter + 36 pp.

[21] L. Katzarkov, M. Kontsevich, T. Pantev, Hodge theoretic aspects of mirror symmetry, From Hodge theory to integrability and TQFT tt*-geometry, Proc. Sympos. Pure Math., 78, Amer. Math. Soc., Providence, RI, 2008, pp. 87-174.

[22] R. Kaufmann, Open/closed string topology and moduli space actions via open/closed Hochschild actions, SIGMA Symmetry Integrability Geom. Methods Appl. 6 (2010), Paper 036, 33 pp.

[23] R. Kaufmann, B. Ward, J. Zuniga, The odd origin of Gerstenhaber brackets, Batalin-Vilkovisky operators, and master equations, J. Math. Phys. 56 (2015), no. 10, 103504, $40 \mathrm{pp}$.

[24] T. Kimura, J. Stasheff, A. A. Voronov, On operad structures of moduli spaces and string theory, Comm. Math. Phys. 171 (1995) 1-25.

[25] T. Kimura, J. Stasheff, A. A. Voronov, Homology of moduli spaces of curves and commutative homotopy algebras, The Gelfand Mathematical Seminars, 19931995, Birkhäuser Boston, 1996, pp. 151-170.

[26] C. Li, S. Li, K. Saito, Primitive forms via polyvector fields, Preprint, arXiv:1311.1659[math.AG],

[27] E. Looijenga, Cohomology of $\mathcal{M}_{3}$ and $\mathcal{M}_{3}^{1}$, Mapping class groups and moduli spaces of Riemann surfaces (Göttingen, 1991/Seattle, WA, 1991), Contemp. Math., 150, Amer. Math. Soc., Providence, RI, 1993, pp. 205-228.

[28] E. Looijenga, Stable cohomology of the mapping class group with symplectic coefficients and of the universal Abel-Jacobi map, Journal of Algebraic Geometry 5 (1996), no. 1, 135-150.

[29] I. Madsen and M. Weiss, The stable moduli space of Riemann surfaces: Mumfords conjecture, Annals of Mathematics (2007), 843-941.

[30] M. Markl, Loop Homotopy Algebras in Closed String Field Theory, Commun. Math. Phys. 221, 367-384 (2001). 
[31] D. Mumford, Abelian quotients of the Teichmüller modular group, Journal d'Analyse Mathématique 18 (1967), no. 1, 227-244.

[32] K. Münster, I. Sachs, Quantum open-closed homotopy algebra and string field theory, Comm. Math. Phys. 321 (2013), no. 3, 769-801.

[33] D. Petersen, Betti numbers of moduli spaces of smooth Riemann surfaces, version: 2012-02-15, https: //mathoverflow . net/q/88515.

[34] O. Randal-Williams, Resolutions of moduli spaces and homological stability, JEMS 18 (2016), no. 1, 1-81.

[35] K. Saito, Moduli space for Fuchsian groups, Algebraic analysis, Vol. II, Academic Press, Boston, 1988, pp. 735-787.

[36] K. Saito, Higher Eichler integrals and vector bundles over the moduli of spinned Riemann surfaces, Prospects in complex geometry (Katata and Kyoto, 1989), Lecture Notes in Math., 1468, Springer, Berlin, 1991, pp. 408-421.

[37] K. Saito, The Teichmller space and a certain modular function from a viewpoint of group representations, Algebraic geometry and related topics (Inchon, 1992), Conf. Proc. Lecture Notes Algebraic Geom., I, Int. Press, Cambridge, MA, 1993, pp. 41-88.

[38] K. Saito, Algebraic representations of Teichmller space, Workshop on Geometry and Topology (Hanoi, 1993), Kodai Math. J. 17 (1994), no. 3, 609-626.

[39] A. Sen, B. Zwiebach, Quantum background independence of closed-string field theory, Nuclear Phys. B 423 (1994), no. 2-3, 580-630.

[40] J. Terilla, Smoothness theorem for differential BV algebras, J. Topol. 1 (2008), no. 3, 693-702.

[41] O. Tommasi, Geometry of Discriminants and Cohomology of Moduli Spaces, Ph.D. thesis, Radboud Universiteit Nijmegen, The Netherlands, 2005, 89 pp., http://hdl.handle.net/2066/27027

[42] A. A. Voronov, Quantizing deformation theory II, Preprint IPMU18-0117, Kavli IPMU, 2018, arXiv:1806.11197 [math.QA], to appear in Pure Appl. Math. Q.

[43] C. Westerland, Configuration spaces in topology and geometry, Gazette of the Australian Mathematical Society, 38 (2011), no. 5, 279-283.

[44] E. Witten, On background-independent open-string field theory, Phys. Rev. D (3) 46 (1992), no. 12, 5467-5473.

[45] B. Zwiebach, Closed string field theory: Quantum action and the BatalinVilkovisky master equation, Nuclear Physics B 390 (1993) 33-152. 
Max Planck Institute for Mathematics, Vivatsgasse 7, 53111 Bonn, GERMANY

E-mail address: sakal008@umn. edu

School of Mathematics, University of Minnesota, Minneapolis, MN 55455, USA, AND KAVLI IPMU (WPI), UTIAS, UNIVERSITY OF TOKYO, KASHIWA, CHIBA 277-8583, JAPAN

E-mail address: voronov@umn.edu 